\title{
Hepatic Niemann-Pick C1-like 1 regulates biliary cholesterol concentration and is a target of ezetimibe
}

\author{
Ryan E. Temel, ${ }^{1}$ Weiqing Tang, ${ }^{1}$ Yinyan Ma, ${ }^{1}$ Lawrence L. Rudel, ${ }^{1}$ Mark C. Willingham, ${ }^{1}$ \\ Yiannis A. Ioannou, ${ }^{2}$ Joanna P. Davies, ${ }^{2}$ Lisa-Mari Nilsson, ${ }^{3}$ and Liqing $\mathrm{Yu}^{1}$ \\ 1Department of Pathology, Section on Lipid Sciences, Wake Forest University School of Medicine, Winston-Salem, North Carolina, USA. \\ 2Department of Human Genetics, Mount Sinai School of Medicine, New York, New York, USA. 'Division of Gastroenterology and Hepatology, \\ Department of Medicine, Karolinska Institute at Karolinska University Hospital Huddinge, Stockholm, Sweden.
}

\begin{abstract}
Niemann-Pick C1-like 1 (NPC1L1) is required for cholesterol absorption. Intestinal NPC1L1 appears to be a target of ezetimibe, a cholesterol absorption inhibitor that effectively lowers plasma LDL-cholesterol in humans. However, human liver also expresses NPC1L1. Hepatic function of NPC1L1 was previously unknown, but we recently discovered that NPC1L1 localizes to the canalicular membrane of primate hepatocytes and that NPC1L1 facilitates cholesterol uptake in hepatoma cells. Based upon these findings, we hypothesized that hepatic NPC1L1 allows the retention of biliary cholesterol by hepatocytes and that ezetimibe disrupts hepatic function of NPC1L1. To test this hypothesis, transgenic mice expressing human NPC1L1 in hepatocytes (L1-Tg mice) were created. Hepatic overexpression of NPC1L1 resulted in a 10- to 20-fold decrease in biliary cholesterol concentration, but not phospholipid and bile acid concentrations. This decrease was associated with a $30 \%-60 \%$ increase in plasma cholesterol, mainly because of the accumulation of apoE-rich HDL. Biliary and plasma cholesterol concentrations in these animals were virtually returned to normal with ezetimibe treatment. These findings suggest that in humans, ezetimibe may reduce plasma cholesterol by inhibiting NPC1L1 function in both intestine and liver, and hepatic NPC1L1 may have evolved to protect the body from excessive biliary loss of cholesterol.
\end{abstract}

\section{Introduction}

Cholesterol is an essential component of cell membranes in mammals. It is required for many important biological processes including synthesis of steroid hormones and bile acids (BAs) and the formation of lipid domains involved in endocytosis and cell signaling. The cholesterol level necessary for normal physiological function can be maintained by de novo synthesis. However, even under conditions of limited availability, cholesterol is lost from the body as a result of hepatobiliary secretion. Because cholesterol synthesis requires substantial energy input, the small intestine has evolved to allow efficient reabsorption of biliary cholesterol. In addition, this absorptive mechanism enables the body to acquire cholesterol from diet.

A recently described protein essential to the body's ability to absorb cholesterol is Niemann-Pick C1-like 1 (NPC1L1) $(1,2)$. Highly expressed on the apical surface of absorptive enterocytes $(1,3,4)$, NPC1L1 is a sterol-sensing domain-containing polytopic transmembrane protein (5) that appears to be the target of ezetimibe, a cholesterol absorption inhibitor. Ezetimibe, commercially known as Zetia, can effectively lower plasma LDL-cholesterol (6-8), presumably through its ability to inhibit NPC1L1 in enterocytes $(1,9)$. However, unlike rodents, in which NPC1L1 is mainly expressed in the intestine, humans highly express NPC1L1

Nonstandard abbreviations used: ABCG5, ABC transporter G5; BA, bile acid; CE, cholesteryl ester; ERL, apoE-rich lipoprotein; FA, fatty acid; FC, free cholesterol; LDLR, LDL receptor; L1-Tg, NPC1L1 transgenic; NPC1L1, Niemann-Pick C1-like 1; PL, phospholipid; RAP, receptor-associated protein; SR-BI, scavenger receptor class B type I; TC, total cholesterol; TG, triglyceride.

Conflict of interest: The authors have declared that no conflict of interest exists. Citation for this article: J. Clin. Invest. 117:1968-1978 (2007). doi:10.1172/JCI30060. in the liver $(1,2,5)$. The exact function of hepatic NPC1L1 remains unknown, but our group has recently shown that NPC1L1 localizes to the canalicular membrane of monkey hepatocytes and that human NPC1L1 expression in hepatoma cells facilitates uptake of free cholesterol (FC) from the culture medium (10). Based upon these data, we hypothesized that, as on the apical membrane of enterocytes, NPC1L1 on the canalicular membrane of hepatocytes may modulate biliary cholesterol excretion by counterbalancing cholesterol efflux mediated by the heterodimeric cholesterol exporter ABC transporter G5 and G8 (ABCG5/ABCG8) $(11,12$ ). The existence of novel mechanisms other than ABCG5/ABCG8 in regulating biliary cholesterol secretion has been suggested based on several recent observations in mice $(13,14)$ and humans $(15)$. Such a mechanism may have evolved to protect the body from excessive loss of cholesterol in the bile. In addition, because ezetimibe and its glucuronated metabolite undergo extensive enterohepatic recirculation $(6,16-20)$, we further hypothesized that ezetimibe inhibits the function of NPC1L1 in the liver. In this study, we created transgenic mice overexpressing human NPC1L1 in the liver. These animal showed a dramatic reduction in biliary cholesterol concentration, which was restored by ezetimibe treatment. Our findings suggest that hepatic NPC1L1 regulates biliary cholesterol excretion and is a target of ezetimibe.

\section{Results}

NPC1L1 is expressed in the liver and localizes to the canalicular membrane in NPC1L1 transgenic mice. To test our hypotheses, 2 independent NPC1L1 transgenic (L1-Tg) mouse lines, L1-Tg20 and L1-Tg112, were created. These mice were fertile and appeared grossly normal. Immunoblot analysis of multiple tissues detected an approxi- 

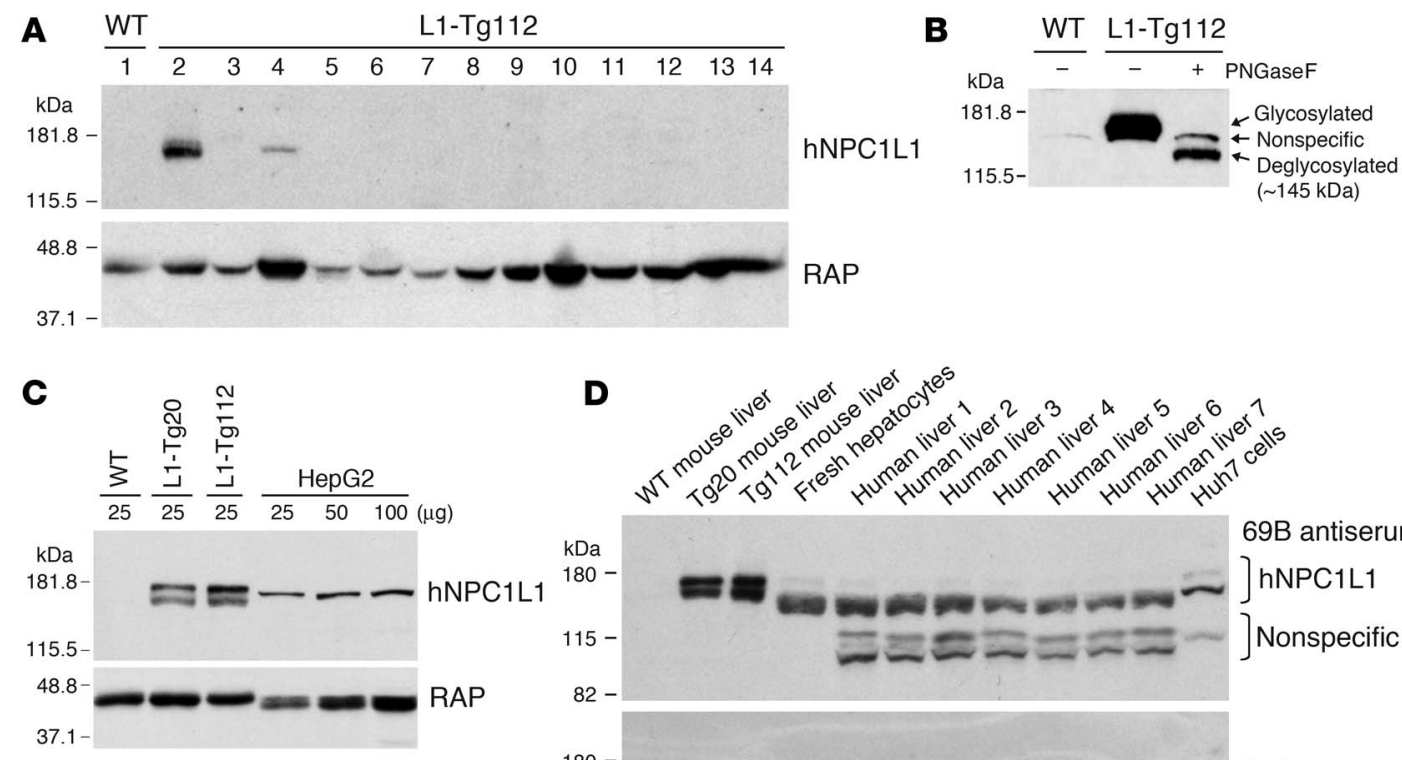

RAP
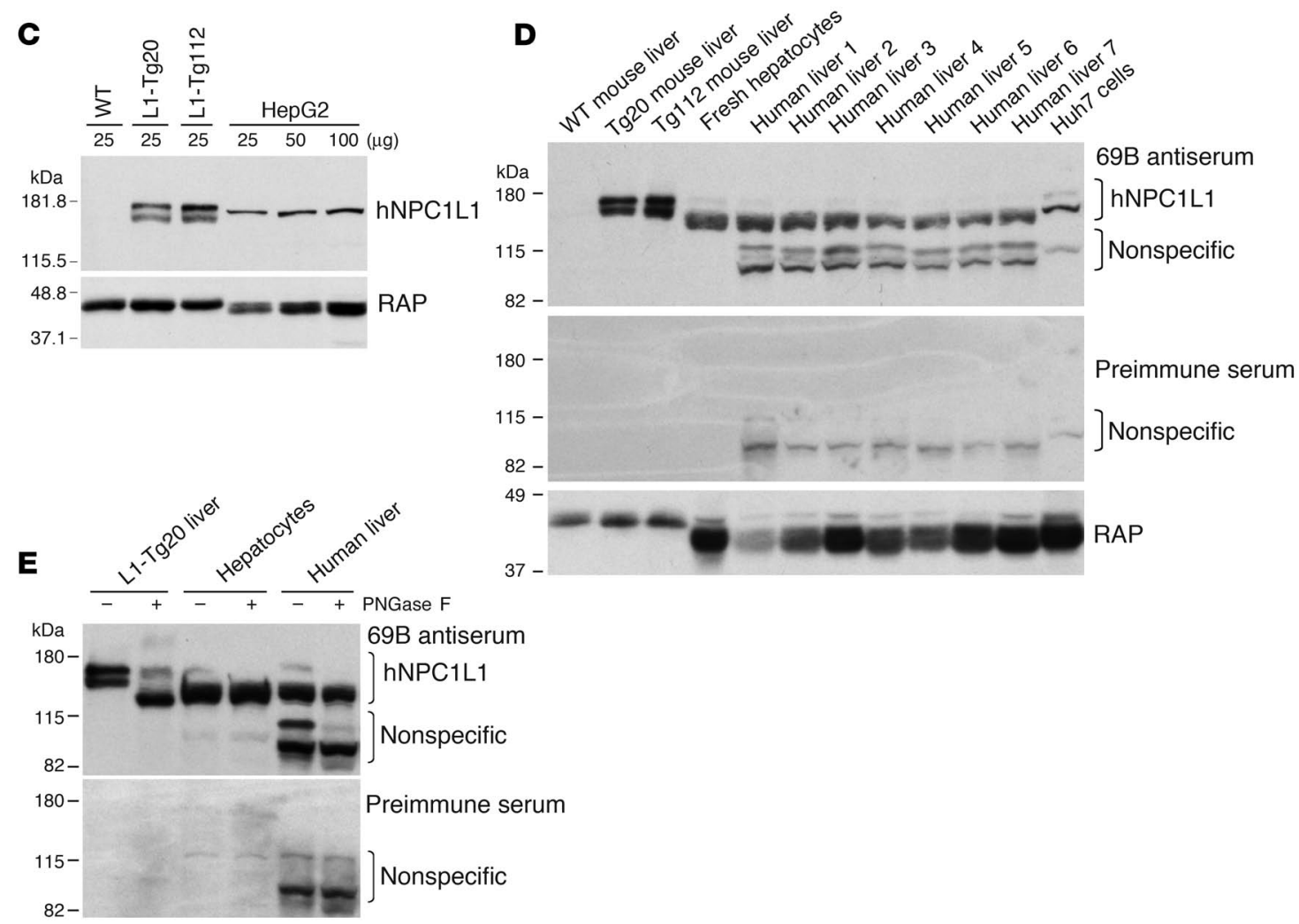

\section{Figure 1}

NPC1L1 is expressed in L1-Tg mouse and human liver tissues. (A) Tissues were collected from 3 male L1-Tg112 mice (3 months old), and equal amounts of tissue from each mouse were pooled and processed for preparation of membrane proteins as described previously (50). Membrane proteins from adrenal glands $(25 \mu \mathrm{g})$ and other tissues $(50 \mu \mathrm{g})$ were fractionated by $8 \%$ polyacrylamide gel in the presence of SDS and immunoblotted with a rabbit anti-human NPC1L1 (anti-hNPC1L1) antibody (L1Ab) (10) and a rabbit anti-rat RAP serum (21). RAP was used as a loading control. Lanes 1 and 2, liver; lane 3, jejunum; lane 4, kidney; lane 5, pancreas; lane 6, lung; lane 7, heart; lane 8, spleen; lane 9, muscle; lane 10, testis; lane 11, cerebrum; lane 12, cerebellum; lane 13, epididymal fat; lane 14, adrenal glands. Similar results were observed in L1-Tg20 mice. (B) Membrane proteins $(50 \mu \mathrm{g})$ from L1-Tg112 mice were treated with or without Peptide:N-glycosidase F (PNGaseF; New England Biolabs) followed by immunoblotting using L1Ab. (C) Mouse liver homogenates and HepG2 cell lysates were immunoblotted with the 69B and RAP antibodies. (D) Mouse liver homogenates $(2.5 \mu \mathrm{g})$ and cell lysates or human liver homogenates $(50 \mu \mathrm{g})$ were immunoblotted with the preimmune serum from the rabbit from which the 69B antiserum was obtained. The same membrane was stripped and immunoblotted with 69B and RAP antibodies. (E) L1-Tg20 mouse liver homogenates $(2.5 \mu \mathrm{g})$ and fresh human hepatocyte lysates or human liver homogenates $(50 \mu \mathrm{g})$ were deglycosylated by Peptide: $N$-glycosidase $\mathrm{F}$, followed by immunoblotting with preimmune serum and $69 \mathrm{~B}$ antiserum.

mately $175-\mathrm{kDa}$ protein only in the livers of L1-Tg mice (Figure 1A). A protein of slightly different size was seen in the kidneys of L1-Tg mice, but was deemed not to be NPC1L1 because it was also observed in the kidneys of WT mice (data not shown). NPC1L1 is highly $N$-glycosylated $(3,10)$. The approximately $175-\mathrm{kDa}$ protein represents the $N$-glycosylated NPC1L1 in L1-Tg livers, because $\mathrm{N}$-glycosidase digestion caused the apparent size of the protein to decrease to about $145 \mathrm{kDa}$ (Figure $1 \mathrm{~B}$ ), the predicted molecular weight of NPC1L1. The relative expression level of human NPC1L1 transgene in the liver of L1-Tg20 and L1-Tg112 mice was about 3.5and 5.7-fold higher, respectively, than that seen in human HepG2 hepatocarcinoma cells, based on densitometry of human NPC1L1 immunoblots of the same amount of whole-tissue homogenates or cell lysates (Figure 1C). NPC1L1 protein was readily detectable from $50 \mu \mathrm{g}$ of fresh human hepatocyte and human liver lysates (Figure 1D). Interestingly, a substantial interindividual variation in hepatic NPC1L1 protein levels was observed in the 7 human liver specimens collected. When the amount of NPC1L1 was normal- 

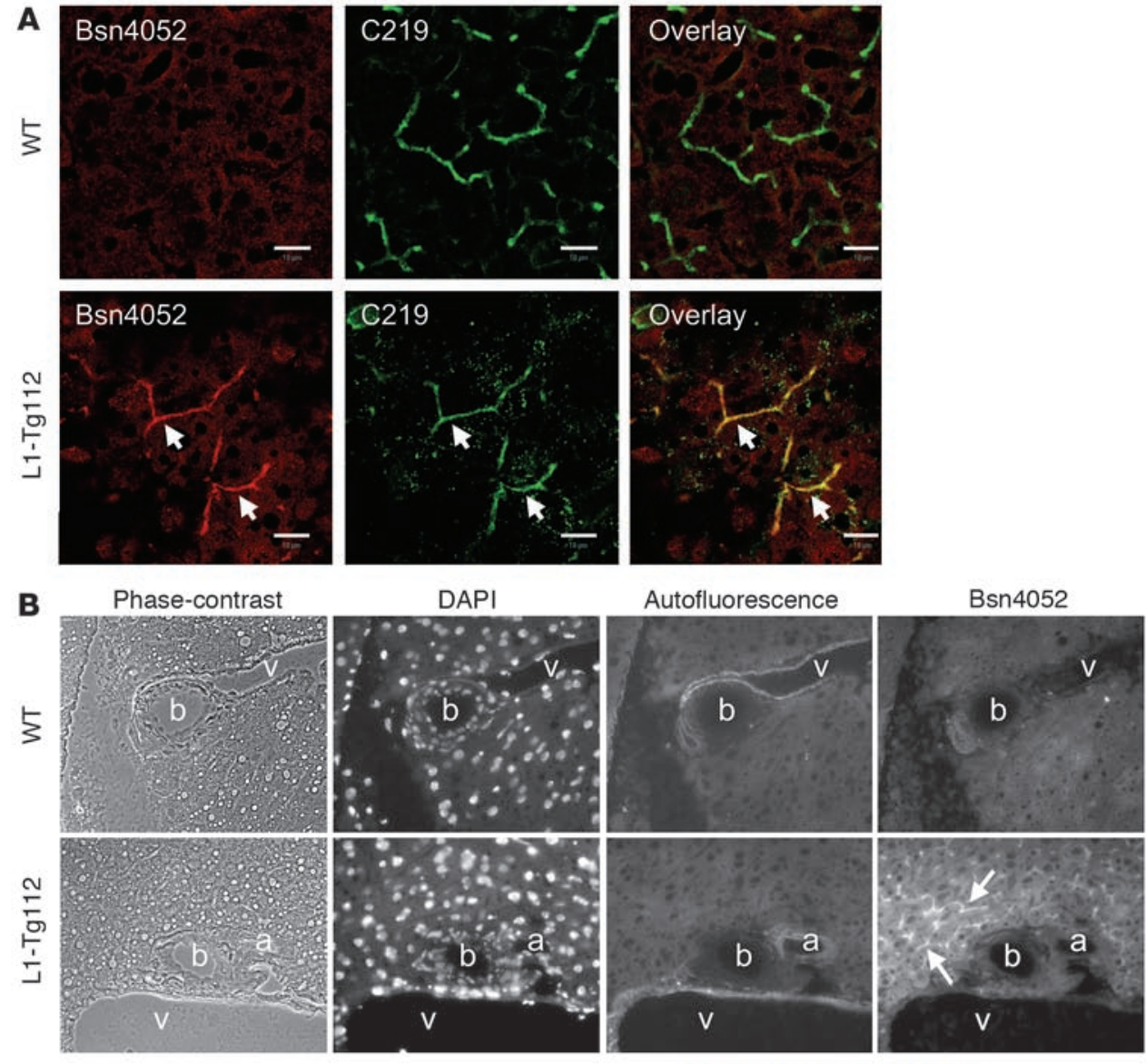

C
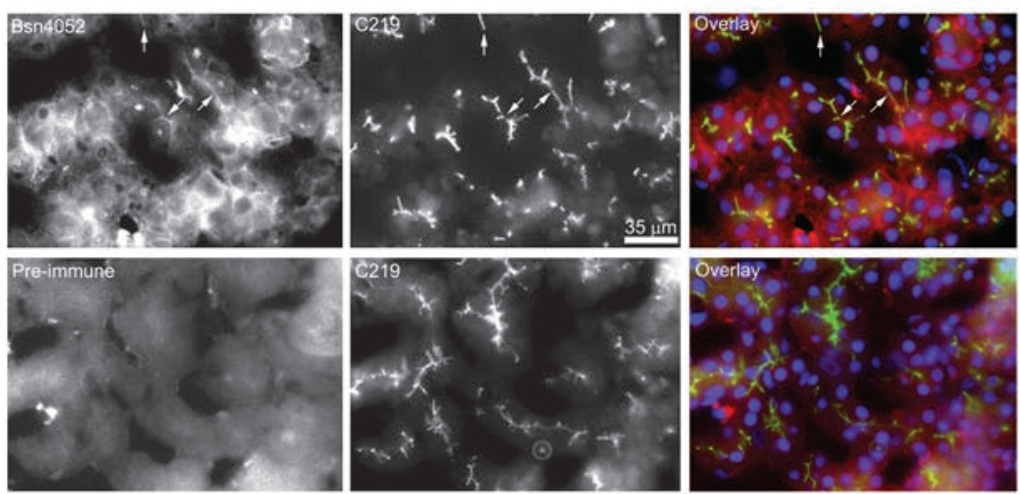

\section{Figure 2}

NPC1L1 localizes to the canalicular membrane in L1-Tg and human liver tissues. (A) Acetone-fixed frozen sections of liver samples from 3-month-old L1-Tg112 and WT male mice fed the $0.015 \%$ cholesterol diet were processed for fluorescence immunohistochemistry with Bsn4052 antibody to human NPC1L1 and mouse monoclonal C219 antibody to ABCB1 as described in Methods. Arrows denote canalicular staining in the section of $\mathrm{L} 1-\mathrm{Tg}$ liver. Original magnification, $\times 630$. Scale bars: $10 \mu \mathrm{m}$. (B) Fluorescence immunohistochemistry of human NPC1L1 was conducted as described in Methods, and sections were examined immediately using an Axioplan 2 fluorescence microscope. Images were taken to show the triad region of liver. Green autofluorescence from elastins in the blood vessel wall was included to distinguish bile duct from blood vessels. Arrows denote canalicular pattern (rhodamine red) of human NPC1L1 staining by Bsn4052 in the L1-Tg liver. Green and red stain appears white. a, artery; b, bile duct; v, vein. Original magnification, $\times 630$. (C) Acetone-fixed frozen sections of human liver specimens were processed for fluorescence immunohistochemistry using C219, Bsn4052, and Bsn4052 preimmune serum as described in Methods, and sections were examined using an Axioplan 2 fluorescence microscope. Arrows denote canalicular pattern of human NPC1L1 staining by Bsn4052 and its colocalization with $A B C B 1$ stained by C219. Scale bars: $35 \mu \mathrm{m}$. ized by a glycoprotein, the receptor-associated protein (RAP) (21), its level in the L1-Tg20 liver was about $86 \%$ of that in human liver specimen 1 (Figure 1D); however, when the NPC1L1 protein was normalized by the protein concentration used for immunoblotting, its level was about 20-fold higher in the L1-Tg20 liver versus human liver specimen 1 . Slight differences in the size of NPC1L1 protein were observed among different tissues and cells (Figure 1D), which may represent differential glycosylation of NPC1L1 in these tissues and cells because $N$-glycosidase digestion caused the protein from mouse and human samples to shift to the similar position (Figure 1E). The extent of $N$-glycosylation of NPC1L1 may be less in human liver than in rodent liver. This may explain why NPC1L1 protein size was smaller in human liver tissue than in mouse livers (Figure 1D). The invisible shift of the strong lower band of NPC1L1 protein in the human liver tissue (Figure 1E) may suggest that a majority of NPC1L1 was slightly $N$-glycosylated in the human liver tissue collected. When a large protein is slightly glycosylated, deglycosylation will not cause a visible shift of the protein size. The smaller size of human versus rodent RAP was consistent with a previous report (22).

Immunofluorescence studies showed that NPC1L1 colocalized with a canalicular P-glycoprotein, ABCB1 (Figure 2A), demonstrating its canalicular location in the L1-Tg liver. No NPC1L1 staining was seen in the WT liver. Additionally, NPC1L1 did not show colocalization with cholangiocytes lining bile ducts (Figure 2B), indicating that NPC1L1 expression is hepatocyte specific. NPC1L1 was also found to localize to the canalicular membrane in human liver by fluorescent immunohistocytochemistry (Figure 2C). 
A

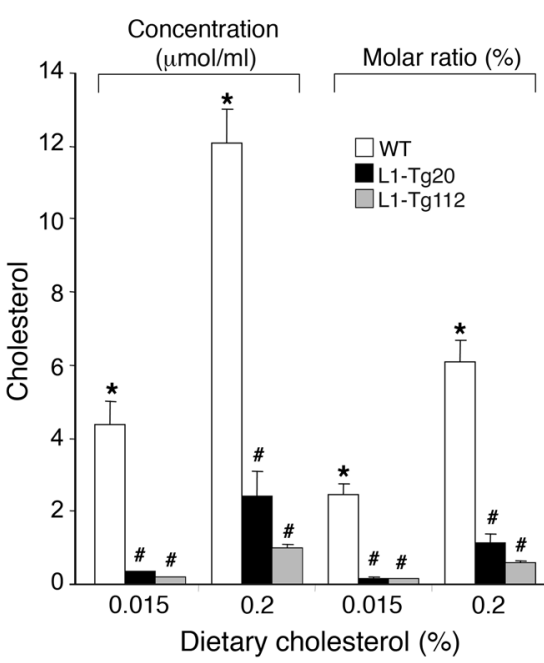

B

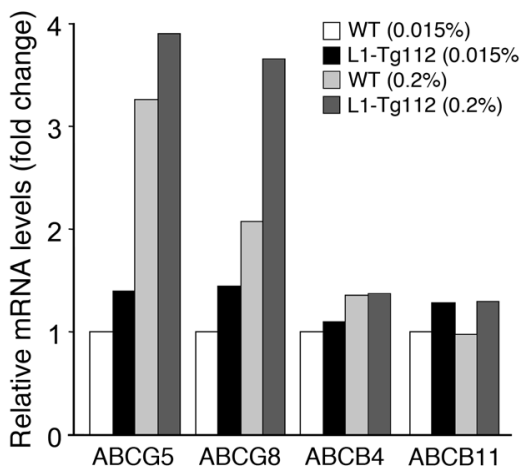

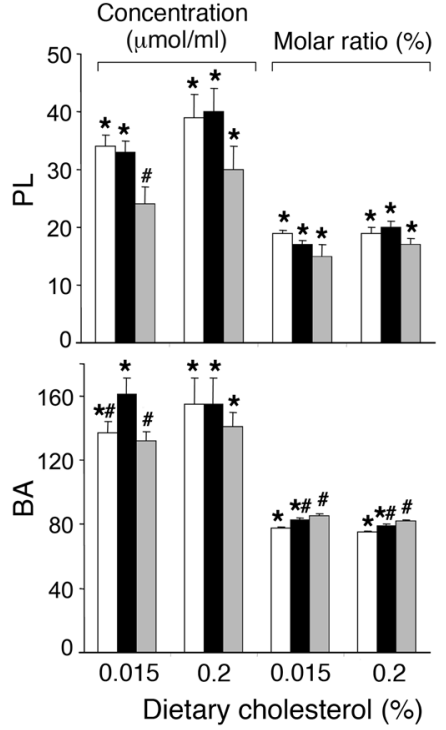

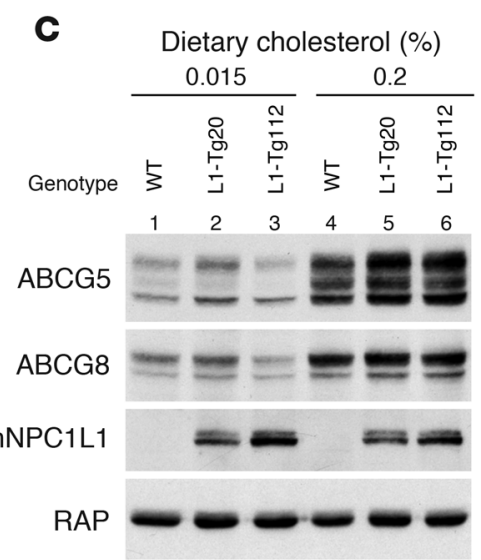

Figure 3

Hepatic expression of NPC1L1 results in a dramatic reduction in biliary cholesterol but not PL and BA. (A) Concentration of gallbladder cholesterol, $\mathrm{PL}$, and $\mathrm{BA}$ in male mice was determined as described in Methods. Molar ratios of each lipid in total biliary lipids were calculated from lipid concentrations. Data are mean \pm SEM of 4-6 samples. (B and $\mathbf{C}$ ) Liver total mRNAs or membrane proteins were prepared as described previously (50) from mice in $\mathbf{A}$, and equal amounts of mRNA or protein from each mouse within a group were pooled ( $n=5$ per group). Levels of selected mRNAs and proteins were determined by quantitative real-time PCR (B) and by immunoblotting (C), respectively, as described previously $(10,50)$. RAP was used as the loading control, and human NPC1L1 was immunoblotted with L1Ab for genotype validation. The experiment was repeated twice with different sets of animals, and similar results were obtained. $P<0.05$ between * and ${ }^{\#}$ groups for each measurement (ANOVA).
Biliary cholesterol is selectively reduced in L1-Tg mice. To test the hypothesis that hepatic NPC1L1 modulates biliary cholesterol excretion, lipid compositions of gallbladder bile were measured in L1-Tg mice (Figure 3A). When fed a $0.015 \%$ cholesterol diet, L1-Tg20 and L1-Tg112 mice showed 13-fold and 23-fold decreases, respectively, in gallbladder cholesterol concentrations compared with WT mice, while phospholipid (PL) and BA concentrations were largely maintained. Feeding a $0.2 \%$ versus $0.015 \%$ cholesterol diet resulted in an increase in gallbladder cholesterol concentrations for mice of all genotypes. However, the cholesterol concentration of L1-Tg20 and L1-Tg112 mice was still 5- and 12-fold lower, respectively, than that of WT mice. No significant differences in gallbladder PL or BA concentration were seen on the $0.2 \%$ cholesterol diet. Because bile can be concentrated in the gallbladder, potentially altering lipid concentrations, molar ratios of biliary lipids were calculated. The molar percentages of biliary cholesterol of L1-Tg20 and L1-Tg112 mice were 14- and 19-fold lower on the $0.015 \%$ cholesterol diet and 5 - and 10 -fold lower on the $0.2 \%$ cholesterol diet, respectively, versus WT mice. L1-Tg mice had slightly higher molar percentages of BA and unchanged molar ratios of PL on both diets compared with WT mice.

Because lack of ABCG5 and/or ABCG8 disrupts biliary cholesterol secretion $(11,23-25)$, the possibility existed that NPC1L1 expression was indirectly changing biliary cholesterol content by decreasing hepatic levels of these proteins. Thus, hepatic mRNA and protein levels of ABCG5 and ABCG8 were determined in mice fed either the $0.015 \%$ or $0.2 \%$ cholesterol diet. On both diets, the mRNA levels of ABCG5 and ABCG8 were slightly higher in L1-Tg112 versus WT mice (Figure $3 B$ ). While the amounts of ABCG5 and ABCG8 proteins remained unchanged on the $0.015 \%$ cholesterol diet, ABCG5 protein appeared to be modestly increased in L1-Tg liver compared with WT liver on the $0.2 \%$ cholesterol diet (Figure 3C). Regardless of genotype, consumption of the $0.2 \%$ cholesterol diet resulted in the expected increase in hepatic ABCG5 and ABCG 8 mRNAs and proteins compared with the $0.015 \%$ cholesterol diet because the 2 genes are targets of liver $X$ receptor (LXR) (26). Thus, the observed reduction in biliary cholesterol of L1-Tg mice was not coupled to decreased hepatic expression of ABCG5 and ABCG8. Because ABCB4 and ABCB11 also alter biliary cholesterol secretion $(27,28)$, mRNA levels of $\mathrm{ABCB} 4$ and $\mathrm{ABCB} 11$ were measured, and no appreciable changes were found (Figure $3 \mathrm{~B}$ ).

Hepatic cholesterol content is minimally affected in L1-Tg mice. To probe the metabolic consequence of NPC1L1-mediated decrease in biliary cholesterol excretion, hepatic and plasma lipids were analyzed. No differences were detected among the $0.015 \%$ cholesterol-fed WT, L1-Tg20, and L1-Tg112 mice in the hepatic content of FC, triglyceride (TG), and PL while the cholesteryl ester (CE) content in L1-Tg20 mice was slightly but significantly higher than in WT 


\section{Table 1}

Hepatic and plasma parameters of WT and L1-Tg mice

\begin{tabular}{|c|c|c|c|c|c|c|}
\hline \multirow[b]{2}{*}{ Genotype } & \multicolumn{3}{|c|}{$0.015 \%$ Dietary cholesterol } & \multicolumn{3}{|c|}{$0.2 \%$ Dietary cholesterol } \\
\hline & WT & L1-Tg20 & L1-Tg112 & WT & L1-Tg20 & L1-Tg112 \\
\hline Hepatic FC (mg/g wet wt) & $2.4(0.1)^{\mathrm{A}}$ & $2.5(0.1)^{A}$ & $2.4(0.1)^{\mathrm{A}}$ & $3.3(0.1)^{\mathrm{A}}$ & $4.0(0.1)^{B}$ & $3.9(0.1)^{\mathrm{B}}$ \\
\hline Hepatic CE (mg/g wet wt) & $1.2(0.2)^{\mathrm{A}}$ & $1.9(0.2)^{B}$ & $1.2(0.1)^{\mathrm{A}}$ & $17(1)^{A}$ & $25(2)^{A}$ & $22(3)^{A}$ \\
\hline Hepatic PL (mg/g wet wt) & $22(0.5)^{\mathrm{A}}$ & $24(0.4)^{\mathrm{A}}$ & $24(0.8)^{A}$ & $21(0.3)^{A}$ & $24(0.5)^{\mathrm{B}}$ & $23(0.4)^{\mathrm{B}}$ \\
\hline Hepatic TG (mg/g wet wt) & $38(4)^{A}$ & $59(14)^{A}$ & $56(8)^{A}$ & $94(14)^{\mathrm{A}}$ & $99(14)^{\mathrm{A}}$ & $112(15)^{A}$ \\
\hline Plasma TC (mg/dl) & $122(4)^{A}$ & $173(13)^{\mathrm{B}}$ & $177(8)^{\mathrm{B}}$ & $154(6)^{A}$ & $205(11)^{\mathrm{B}}$ & $242(16)^{B}$ \\
\hline Plasma FC (mg/dl) & $29(1)^{A}$ & $41(2)^{B}$ & $35(1)^{C}$ & $34(2)^{A}$ & $46(1)^{\mathrm{B}}$ & $40(1)^{C}$ \\
\hline Plasma CE (mg/dl) & $155(5)^{A}$ & $221(20)^{B}$ & $236(12)^{B}$ & $200(8)^{A}$ & $266(17)^{A}$ & $338(26)^{B}$ \\
\hline Plasma PL (mg/dl) & $245(8)^{\mathrm{A}}$ & $332(29)^{\mathrm{B}}$ & $321(12)^{B}$ & $292(14)^{A}$ & $380(19)^{\mathrm{B}}$ & $402(18)^{B}$ \\
\hline Plasma TG (mg/dl) & $89(10)^{A}$ & $75(5)^{A}$ & $81(10)^{A}$ & $75(12)^{A}$ & $94(12)^{A}$ & $66(9)^{A}$ \\
\hline Body weight (g) & $28.6(0.4)^{A}$ & $33.2(1.2)^{\mathrm{A}}$ & $29.4(2.2)^{A}$ & $32.8(1.2)^{\mathrm{A}}$ & $32.8(1.3)^{\mathrm{A}}$ & $31.4(1.3)^{\mathrm{A}}$ \\
\hline Liver weight $(\mathrm{g})$ & $1.2(0.1)^{\mathrm{A}}$ & $1.4(0.1)^{\mathrm{A}}$ & $1.3(0.1)^{A}$ & $1.5(0.1)^{A}$ & $1.6(0.1)^{A}$ & $1.6(0.1)^{A}$ \\
\hline Liver/body weight ratio (\%) & $4.0(0.2)^{\mathrm{A}}$ & $4.3(0.2)^{\mathrm{A}}$ & $4.4(0.2)^{\mathrm{A}}$ & $4.6(0.2)^{\mathrm{A}}$ & $4.8(0.2)^{\mathrm{A}}$ & $5.0(0.2)^{\mathrm{A}}$ \\
\hline AST (U/l) & $44(9)^{A}$ & $28(12)^{A}$ & $56(5)^{A}$ & ND & ND & ND \\
\hline $\operatorname{ALT}(\mathrm{U} / \mathrm{I})$ & $18(3)^{A}$ & $22(2)^{A}$ & $20(2)^{A}$ & ND & ND & ND \\
\hline Bilirubin (mg/dl) & $0.26(0.4)^{\mathrm{A}}$ & $0.24(0.02)^{\mathrm{A}}$ & $0.42(0.2)^{A}$ & ND & ND & ND \\
\hline
\end{tabular}

mice (Table 1). When fed the $0.2 \%$ cholesterol diet, L1-Tg20 and L1-Tg112 mice had modestly but significantly higher amounts of hepatic FC and PL compared with WT mice (Table 1). No differences were found in the hepatic content of CE and TG among L1-Tg20, L1-Tg112, and WT mice fed the $0.2 \%$ cholesterol diet. Thus, the NPC1L1-mediated inhibition of biliary cholesterol excretion only resulted in modest accumulation of cholesterol in the liver. In addition, no differences were observed between WT and L1-Tg mice in body weights, liver weights, liver/body weight ratios, and plasma levels of aspartate aminotransferase, alanine aminotransferase, and bilirubin (Table 1), which indicates that L1-Tg mice grow normally and have normal liver function.

The minimal changes in the hepatic cholesterol concentration of L1-Tg mice was consistent with the minor changes in the mRNA levels of LXR target genes and genes in the cholesterol biosynthetic pathway, including SREBP-1c, PL transfer protein, ABCG5/ ABCG8 (Figure 3B), SREBP-2, HMG coenzyme A reductase, and HMG coenzyme A synthase (data not shown).

Plasma cholesterol is increased in L1-Tg mice. To probe the trafficking of NPC1L1-derived cholesterol, plasma lipids were measured. Interestingly, significant increases in plasma cholesterol concentrations were observed in L1-Tg mice (Table 1). When fed the $0.015 \%$ cholesterol diet, L1-Tg20 and L1-Tg112 mice showed a $43 \%$ increase in plasma total cholesterol (TC) and a $41 \%$ and $21 \%$ increase, respectively, in FC compared with WT mice. Feeding the $0.2 \%$ versus $0.015 \%$ cholesterol diet caused plasma TC and FC to increase for all 3 genotypes. However, L1-Tg20 and L1-Tg112 mice still displayed significantly higher plasma TC and FC concentrations than in WT mice. Plasma TG concentrations were similar for mice of all 3 genotypes on both diets.

Increased plasma cholesterol largely accumulates in apoE-rich HDL in L1-Tg mice. To further characterize the changes in plasma cholesterol concentration, plasma lipoprotein cholesterol and apolipoprotein distributions were determined for L1-Tg112 and WT mice (Figure 4, A and B). On both $0.015 \%$ and $0.2 \%$ cholesterol diets, L1-Tg112 compared with WT mice had increases in HDL-cholesterol. However, the apolipoprotein composition of L1-Tg112 and WT HDL was very similar (Figure 4B; fractions 29-32), with the major constituent being apoA-I and the minor constituents being apoA-II, apoA-IV, and apoCs. In addition to the increase in HDL-cholesterol, L1-Tg112 mice had a dramatic increase in cholesterol carried on lipoproteins with a size indicative of either small LDL or large HDL. Although minor amounts of apoB100, apoA-I, apoA-II, and apoCs were present, the main apolipoprotein on this lipoprotein species in both L1-Tg112 and WT mice was apoE (Figure 4B; fractions 24-26). Because the amount of apoE on the apolipoprotein distribution gel is not quantitative, plasma apoE levels were determined by immunoblotting and densitometry. Compared with WT mice, the plasma apoE protein level was significantly higher in L1-Tg mice (Figure 4C).

To characterize the apoE-rich lipoprotein (ERL), we isolated LDL, ERL, and HDL from WT and L1-Tg112 mice by size exclusion chromatography and determined the percent chemical compositions of these particles as described previously (29). Similar to apolipoprotein compositions (Figure 4B), the percent chemical compositions were nearly identical within each lipoprotein class isolated from WT and L1-Tg112 mice (Table 2). However, percent chemical compositions of LDL, ERL, and HDL were dramatically different. ERL had percentages of FC and protein that were similar to those of LDL. In contrast, the percentages of TG and PL on ERL were like those of HDL. Thus, ERL appeared to have chemical compositions similar to both LDL and HDL. However, compared with LDL and HDL, ERL was unique in that it contained an intermediate amount of $\mathrm{CE}$ and had the lowest $\mathrm{CE} / \mathrm{FC}$ ratio, indicating the enrichment of FC in this lipoprotein particle.

It has been shown that in mice, saturated and monounsaturated fatty acids (FAs), which are predominantly used by acyl-CoA:choles- 


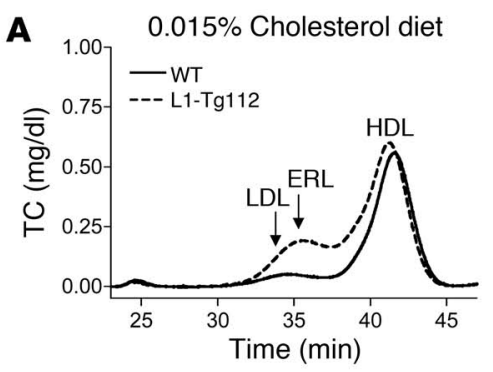

B

WT

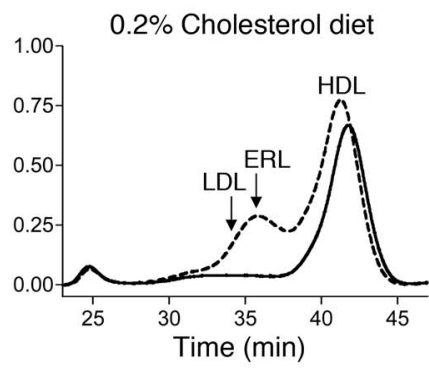

L1-Tg112

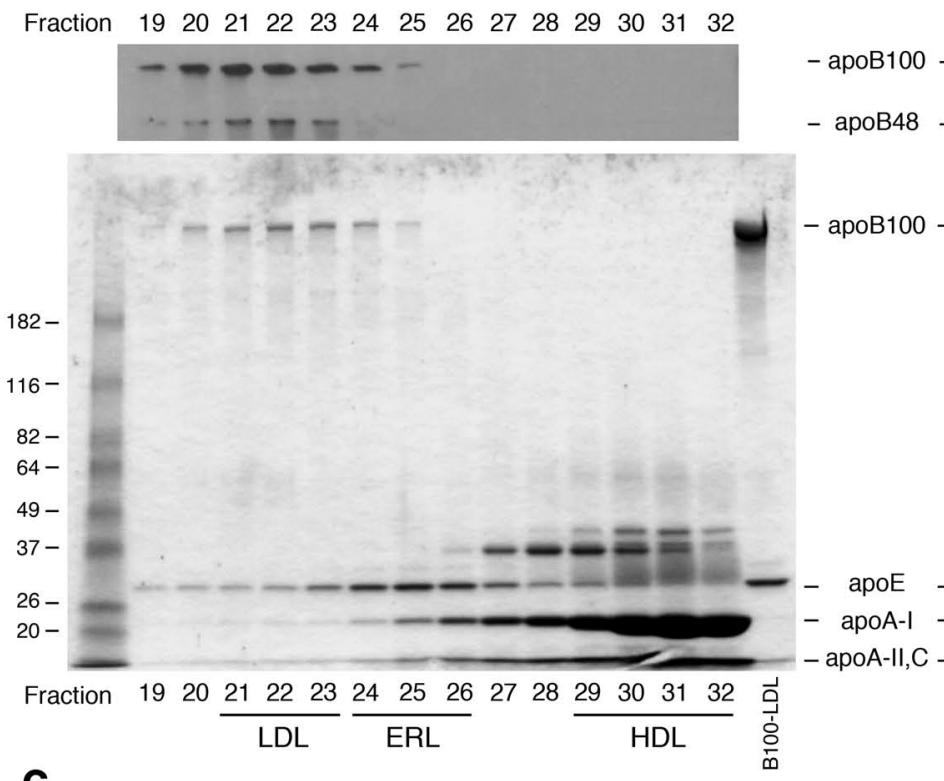

C

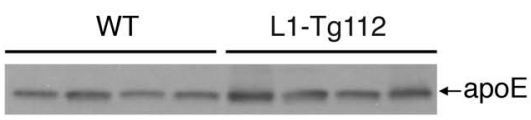

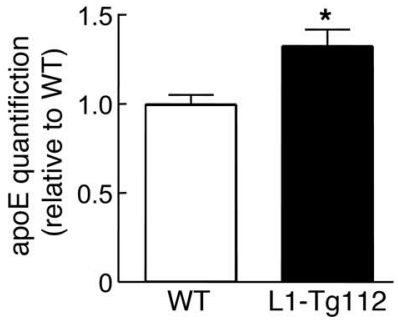

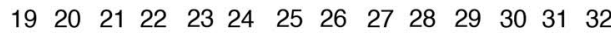

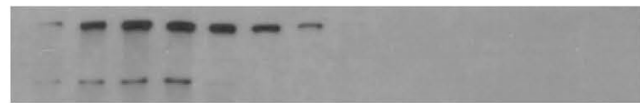

$1920 \frac{2122 \quad 23}{\mathrm{LDL}} \frac{24 \quad 2526}{\mathrm{ERL}} 2728 \frac{293031 \quad 32}{\mathrm{HDL}} \frac{\overrightarrow{1}}{\frac{1}{8}}$

D

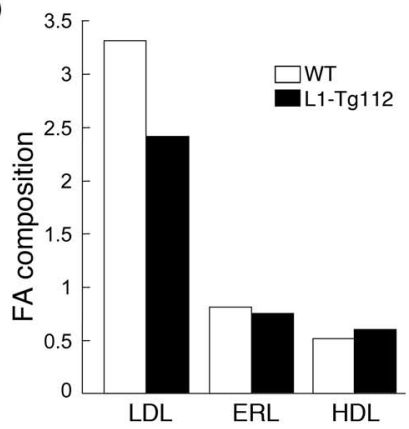

\section{Figure 4}

Increased plasma cholesterol is mainly carried on the apoE-rich HDL. (A) Plasma lipoprotein cholesterol distribution in male WT and L1-Tg112 mice was determined as described in Methods. (B) Apolipoprotein distribution of plasma lipoproteins from male WT and L1-Tg112 mice was determined as described in Methods. Data represent a qualitative, not a quantitative, analysis of apolipoproteins. (C) Quantification of plasma apoE levels by immunoblotting. Plasma was collected from 4 WT and 4 L1-Tg112 mice fed the $0.2 \%$ cholesterol diet for 21 days. After 1:200 dilution in saline, $14 \mu \mathrm{l}$ of diluted samples was immunoblotted with a rabbit anti-rat apoE serum and quantified as described in Methods. Values are mean \pm SEM of 4 samples. (D) FA composition of lipoprotein CE was determined as described in Methods in male WT and L1-Tg112 mice. Values are the sum of the percentages of saturated (16:0 and 18:0) and monounsaturated (16:1 and 18:1) FAs divided by the percentage of polyunsaturated (18:2, 20:4, 20:5, and 22:6) FAs. ${ }^{*} P<0.05$ versus WT (Student's $t$ test).

terol acyltransferase 2 (ACAT2) in liver, are the major FAs in LDL$\mathrm{CE}$, while polyunsaturated FAs, which are preferentially used by lecithin:cholesterol acyltransferase (LCAT) in plasma, represent the bulk of FAs in HDL-CE $(30,31)$. To further define ERL, the FA composition of CE in ERL was determined. The ratios of saturated plus monounsaturated FA to polyunsaturated FA for LDL-CE, ERL-CE, and HDL-CE were approximately $3,0.8$, and 0.6 , respectively (Figure
4D). These results indicate that the FA composition of ERL-CE is similar to that found in HDL-CE, which is mainly LCAT derived. Thus, we designated these lipoprotein particles "apoE-rich HDL."

Because large apoE-rich HDL particles are also observed in mice lacking scavenger receptor class B type I (SR-BI) $(32,33)$ and in transgenic mice overexpressing ABCA1 in the liver (34), hepatic expression of SR-BI and ABCA1 were determined. Regardless 


\section{Table 2}

Chemical composition of plasma lipoproteins

\begin{tabular}{lccccccc} 
& & FC (\%) & CE (\%) & TG (\%) & PL (\%) & Protein (\%) & CE/FC ratio \\
WT & LDL & 11 & 39 & 6 & 22 & 21 & 3.5 \\
L1-Tg112 & LDL & 12 & 41 & 2 & 23 & 22 & 3.5 \\
WT & ERL & 14 & 31 & 1 & 30 & 23 & 2.2 \\
L1-Tg112 & ERL & 13 & 33 & 0 & 31 & 23 & 2.6 \\
WT & HDL & 4 & 23 & 0 & 29 & 44 & 5.9 \\
L1-Tg112 & HDL & 3 & 24 & 0 & 28 & 45 & 7.8 \\
\hline
\end{tabular}

Plasma was isolated from 4-hour-fasted male WT and L1-Tg112 mice fed the $0.2 \%$ cholesterol diet for 21 days. Following isolation by density ultracentrifugation, plasma lipoproteins were fractionated by size exclusion chromatography, and fractions representing LDL (fractions 21-23), ERL (fractions 24-26), and HDL (fractions 29-33) were pooled. Total lipids were extracted, and enzymatic assays were used to determine concentrations of TC, FC, TG, and PL. CE concentrations were calculated by multiplying the mass difference between TC and FC by 1.67. Protein concentrations for the samples were determined using a Lowry assay.

was not significantly different from that of either vehicle-treated or ezetimibe-treated WT mice.

\section{Discussion}

In 2 independent transgenic mouse lines, we found that the canalicular expression of NPC1L1 in the liver resulted in a drastic reduction in biliary cholesterol concentration, but not PL and BA concentrations; this decrease was associated with an increase in plasma cholesterol concentration. Increased plasma cholesterol was mainly associated with apoE-rich HDL particles. The cholesterol absorption inhibitor ezetimibe virtually returned the phenotype of L1-Tg mice to normal. These findings define the hepatic NPC1L1 pathway as a potential mechanism regulating whole-body cholesterol homeostasis and the liver as another target of ezetimibe action.

The finding of canalicular localization of NPC1L1

of genotype and cholesterol feeding, hepatic mRNA levels of $S R-B I$ and $A B C A 1$ were largely unaffected (data not shown). SR-BI protein levels also remained unchanged (Figure 5). Interestingly, ABCA1 protein levels were consistently higher in L1-Tg mice compared with WT mice fed either diet (Figure 5). When compared with the mice fed the $0.015 \%$ cholesterol diet, both WT and $\mathrm{L} 1-\mathrm{Tg}$ mice fed the $0.2 \%$ cholesterol diet had higher ABCA1 protein levels. To further explore the mechanism underlying cholesterol accumulation in the plasma of L1-Tg mice, the hepatic mRNA levels of other genes important in lipoprotein metabolism - including $L D L$ receptor $(L D L R), a p o B, a p o E$, and proprotein convertase subtilisin/kexin type 9a (PCSK9) - were also quantified, and no changes were found (data not shown). The protein level of LDLR was also determined, and no changes were observed (Figure 5).

Ezetimibe restores biliary cholesterol excretion in L1-Tg mice. To test our hypothesis that ezetimibe inhibits hepatic NPC1L1 function, the effect of ezetimibe on biliary cholesterol excretion was determined in WT and L1-Tg20 mice treated with either vehicle or 10 $\mathrm{mg}$ ezetimibe $/ \mathrm{kg} / \mathrm{d}$ for 4 days. Bile was collected by cannulation of the common bile duct, and its lipid composition was measured (Figure 6A). Based upon molar ratio, 10-fold less cholesterol was found in the bile of vehicle-treated L1-Tg20 versus WT mice. However, the molar ratio of biliary cholesterol in ezetimibe-treated L1-Tg20 mice was similar to that of ezetimibe-treated WT mice. Results were similar for biliary cholesterol concentrations. Unlike cholesterol, biliary PL and BA concentrations and molar ratios were not different in WT and L1-Tg20 mice treated with either vehicle or ezetimibe. Furthermore, it was determined that ezetimibe increased fecal neutral sterol (cholesterol and its bacterial metabolites) excretion in both WT and L1-Tg20 mice (Figure 6B), thus demonstrating that the effect of ezetimibe on intestinal cholesterol absorption was maintained in L1-Tg mice.

Ezetimibe restores plasma cholesterol concentration in L1-Tg mice. To examine the effect of ezetimibe on plasma cholesterol metabolism, plasma TC was measured in mice treated with either vehicle or ezetimibe $(10 \mathrm{mg} / \mathrm{kg} / \mathrm{d})$ for 7 days (Figure 6C). When treated with vehicle, L1-Tg112 mice maintained a significant 38\% increase in plasma TC compared with WT mice. However, administration of ezetimibe to L1-Tg112 mice caused the TC concentration to significantly decrease by $26 \%$ compared with vehicle-treated L1-Tg112 mice. In addition, the TC concentration of ezetimibe-treated L1-Tg112 mice in L1-Tg and human liver is consistent with our previous studies showing that NPC1L1 localizes to the canalicular membrane of hepatocytes in monkeys and on the canalicular-like structure of cultured hepatoma cell couplets (10). It also corresponds to the concentration of rat NPC1L1 to the apical membrane of enterocytes $(1,3)$. Therefore, we believe that the canalicular membrane of hepatocytes represents the natural location of NPC1L1 in liver.

One reasonable explanation for the reduction in biliary cholesterol in L1-Tg mice is that canalicular NPC1L1 transports cholesterol from the canalicular bile back into hepatocytes. Several pieces of evidence support this explanation: (a) NPC1L1 is required for enterocytes to absorb FC from the intestinal lumen (1), which indicates that the protein is in the cholesterol uptake pathway; (b) our recent study shows that NPC1L1 expression in hepatoma cells facilitates the uptake of extracellular FC without inhibiting cholesterol efflux (10); (c) NPC1L1 physically localizes to the canalicular membrane of hepatocytes (10) (Figure 2); and

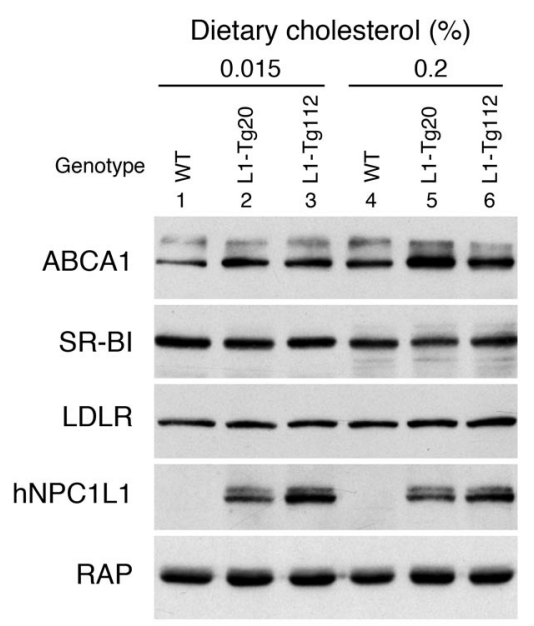

Figure 5

Hepatic ABCA1 protein is increased in L1-Tg mice. The immunoblotting filter used in Figure $2 \mathrm{C}$ was stripped and reblotted with the antibodies to ABCA1 (51), SR-BI (46), and LDLR (46). The immunoblot of human NPC1L1 and RAP shown in Figure $3 \mathrm{C}$ are reproduced here as a control. The experiment was repeated twice with similar results. 
A
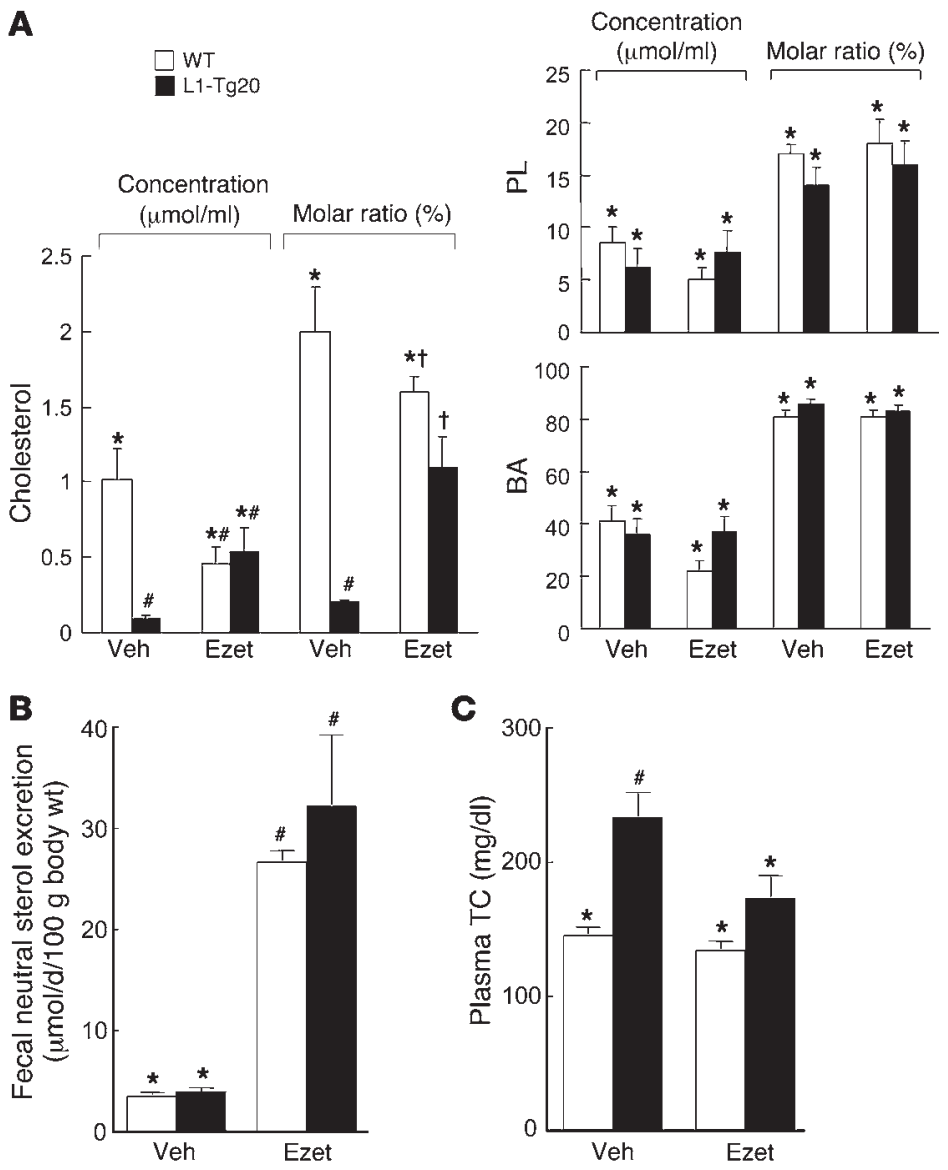

c

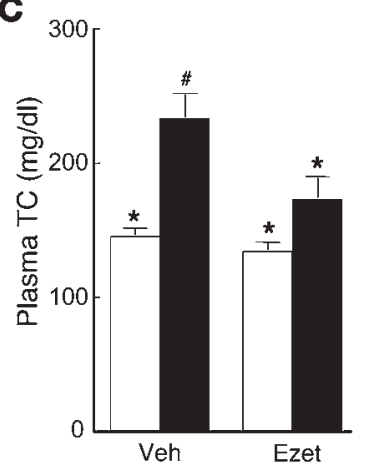

\section{Figure 6}

Ezetimibe restores biliary and plasma cholesterol concentrations in L1-Tg mice. (A) Bile was collected by common bile duct cannulation from male WT and L1-Tg20 mice treated with ezetimibe (Ezet) as described in Methods. Biliary lipid concentrations were analyzed, and lipid molar ratios were calculated. Veh, vehicle. (B) Fecal neutral sterol excretion was measured in WT and L1-Tg20 mice treated with ezetimibe as described in Methods. (C) After being fed the $0.015 \%$ cholesterol diet for 18 days, male WT and L1-Tg112 mice were gavaged daily on days $18-24$ with either $10 \mathrm{mg} / \mathrm{kg}$ ezetimibe or vehicle. Mice were maintained on the same diet during the treatment. After a 4-hour fast on day 25, mice were sacrificed, and plasma TC concentration was determined. Values are mean \pm SEM of 4-5 (A and B) or 5-7 (C) samples. $P<0.05$ among * , , and ${ }^{\dagger}$ groups for each measurement (ANOVA).

ABCG5/ABCG8 protein levels (Figure 3C). Further studies are required to address these questions.

The increased amount of cholesterol found in normalsized HDL and large apoE-rich HDL could be a result of the cholesterol that NPC1L1 retains in the liver stimulating ABCA1-dependent HDL formation. Consistent with this, the protein level of ABCA1 is higher in the $\mathrm{L} 1-\mathrm{Tg}$ liver (Figure 5), and liver-specific ABCA1 transgenic mice display increases in plasma TC and apoE-rich HDL (34). It remains possible, however, that the hepatic expression of NPC1L1 is disrupting the clearance of apoE-rich HDL-cholesterol by the liver. ApoE-rich HDL accumulates in SR-BI-deficient mice because of the liver's inability to efficiently remove HDL-cholesterol from the circulation $(32,33)$. However, our finding of normal SR-BI expression (Figure 5) suggests

(d) recent reports suggest that mechanisms other than ABCG5/ ABCG8 participate in regulating biliary cholesterol secretion in mice $(13,14)$ and humans $(15)$. However, directly measuring cholesterol uptake from the canalicular side of a hepatocyte in an animal is technically challenging. In order to measure cholesterol uptake from the bile canaliculus, labeled cholesterol, together with a nonabsorbable marker, has to be precisely delivered to the microscopic bile canaliculus, followed by the measurement of the labeled cholesterol and the marker in isolated hepatocytes and plasma. Introducing labeled cholesterol and a marker retrograde from the bile duct to the bile canaliculus requires certain pressure to be applied to the lumen of bile duct to counter the force of constant bile flow, which may damage the tight junction between hepatocytes and cause a leak of the labeled cholesterol and the marker into the liver and plasma. Additionally, sitosterol and sitostanol, the 2 nonabsorbable sterol markers that have been used to measure intestinal cholesterol absorption, could not be used in this situation because they likely share with cholesterol a common NPC1L1 route for entering a cell (4). As a result of technical difficulties, no direct evidence has been available in support of a mechanism transporting cholesterol from the canalicular bile into hepatocytes. As an apical transporter for cholesterol uptake (1), NPC1L1 is a good candidate for this mechanism. However, another possibility is that NPC1L1 expression in the L1-Tg liver partitions cholesterol away from the pool that is normally delivered to ABCG5/ABCG8 for biliary secretion. Additionally, NPC1L1 may interfere with the cholesterol transporting activity of ABCG5/ABCG8 without affecting that this HDL receptor may not play a critical role in the accumulation of apoE-rich HDL in L1-Tg mice. Intriguingly, the apoE-rich HDL particles accumulated in L1-Tg mice were enriched in FC, which is very similar to the cholesterol-induced apoE HDLc found in cholesterol-fed swine and dogs $(35,36)$. ApoE HDLc was reported to be functionally similar to LDL, with a much higher binding affinity to $\operatorname{LDLR}(37,38)$. The canine apoE HDLc was found to be rapidly cleared from the plasma of rats and dogs and almost quantitatively recovered in the liver within minutes of injection $(39,40)$. Its kinetic uptake properties are almost identical to rat chylomicron remnants (36). It will be instructive to study the in vivo kinetics and LDLR-binding affinity of apoE-rich HDLs from L1-Tg mice in the future.

The near restoration of plasma and biliary cholesterol concentrations in ezetimibe-treated L1-Tg mice demonstrated that the amount of transgene-expressed NPC1L1 proteins in L1-Tg liver was at a pharmacologically inhibitable level. It also indicates that ezetimibe can block the function of NPC1L1 not only in enterocytes but also in hepatocytes. The gallbladder cholesterol concentration increased 2- to 4-fold in dogs treated with ezetimibe (41), which may be a result of inhibition of hepatic NPC1L1 by ezetimibe. Interestingly, we have detected a protein the size of NPC1L1 in $50 \mu \mathrm{g}$ of dog liver homogenates that specifically immunoreacted with the 69B rabbit anti-human NPC1L1 serum but not the preimmune serum from the same rabbit (data not shown), which suggests that dog liver may express NPC1L1. If NPC1L1 in human liver does indeed facilitate the reuptake of cholesterol from canalicular bile, ezetimibe may inhibit this function of hepatic 
NPC1L1. This action of ezetimibe may allow a greater amount of biliary cholesterol to be excreted from the liver, thus stimulating the final step in the reverse cholesterol transport pathway. On the other hand, because increase in biliary cholesterol secretion may cause gallstone formation, the use of ezetimibe in individuals predisposed to gallstone disease should be evaluated. Alternatively, ezetimibe inhibition of hepatic NPC1L1 may not increase gallstone formation because the drug simultaneously blocks biliary and dietary cholesterol absorption in the small intestine, making less cholesterol available for biliary secretion.

The hepatic function of NPC1L1 may be to protect the body from excessive biliary loss of cholesterol. Alternatively, NPC1L1 may be expressed on the canalicular membrane to more finely regulate the membrane cholesterol content in the face of net hepatic cholesterol secretion into bile. The presence of both efflux and import mechanisms on the same membrane is an example of a dual or push-pull mechanism of dynamic regulation and enables more precise regulation of membrane cholesterol content with lower intrinsic noise (42). There is emerging evidence that membrane cholesterol content is the crucial factor in resistance of the canalicular membrane against the cytotoxic effects of bile salts (43). Several lines of evidence suggest that sphingomyelin and cholesterol are major constituents of the outer leaflet of the canalicular membrane, where they would promote the formation of a rigid "liquid-ordered" phase. In contrast to more fluid liquid crystalline membranes, a rigid cholesterol and sphingomyelin-rich membrane would be relatively resistant to the detergent effects of the millimolar concentrations of BAs in the canalicular space. The bile salt composition is considerably more hydrophobic in humans than in mice, with considerable amounts of chenodeoxycholate and deoxycholate, dihydroxy bile salts that are potent detergents. In contrast, the pool in mice consists largely of the hydrophilic bile salts cholate and muricholate, and mice are able to efficiently rehydroxylate more hydrophobic secondary BAs that are reabsorbed from the intestine. Whereas the lipid phase of the canalicular membrane may be less important for protection against the hydrophilic bile salts present in mice, humans may have evolved to express higher levels of hepatic NPC1L1 in order to finely control the canalicular cholesterol content and protect against hepatic injury and cholestasis.

\section{Methods}

Creation of L1-Tg mice. A full-length NPC1L1 cDNA (GenBank accession no. AY436875) was cloned as described previously (10). For the creation of L1-Tg mice, $\mathrm{KpnI}$ and ClaI sites were added to $5^{\prime}$ and $3^{\prime}$ ends, respectively, of NPC1L1 cDNA. This CDNA was then cloned into the HpaI/ClaI restriction sites of the pLiv-11 construct (44) after blunting the KpnI site with T4 DNA polymerase. The pLiv-11 construct was chosen because it has been used previously by other investigators to direct transgene expression in liver $(34,45)$. Because the SalI site in the pLiv-11 construct cannot be used to release the transgene cassette as a result of the presence of this site in NPC1L1 cDNA, it was engineered and replaced by a cluster of rare restriction sites (Srfl, PmeI, NotI, and PacI) before cDNA cloning. The transgene cassette including human apoE promoter, NPC1L1 cDNA, and human apoE hepatic control region was released from the construct by NotI/SpeI digestion. After purification, the transgene was microinjected into the fertilized embryos of B6D2 F1 mice (Harlan) in the Transgenic Mouse Core Facility of Wake Forest University Health Sciences, and transgenic mouse lines L1-Tg20 and L1-Tg112 were established by implanting microinjected embryos into foster mothers. The transgene cassette was found to be intact in these lines by PCR screening of different regions. Mendelian inheritance of transgene was confirmed by $50 \%$ positive offspring from each transgenic founder. Hemizygous positive L1-Tg mice were always crossed to WT B6D2 F1 mice to generate hemizygous L1-Tg mice and WT littermate controls for all experiments.

All mice were housed in a specific pathogen-free animal facility in plastic cages in a temperature-controlled room $\left(22^{\circ} \mathrm{C}\right)$ with a 12-hour light/ 12 -hour dark cycle. The mice were fed ad libitum a cereal-based rodent chow diet unless stated otherwise and had free access to water. All animal procedures were approved by the institutional animal care and use committee at Wake Forest University Health Sciences.

Collection of human tissue. Normal liver tissue was obtained from patients referred to Karolinska University Hospital Huddinge for surgical liver resection due to cancer. The liver specimens were frozen in liquid nitrogen and stored at $-70^{\circ} \mathrm{C}$ for future use. The study with human liver tissue was approved by the Human Ethics Committee of Karolinska Institute. Written informed consent was given by the individual patients.

Preparation and Western analysis of tissue homogenates and cell lysates. A total of 50-100 mg of tissue sample was homogenized in $0.5 \mathrm{ml}$ lysis buffer $(20$ mM HEPES-HCl, pH 7.4; 5 mM MgCl $; 1$ mM EGTA; $10 \%$ glycerol; $1 \%$ Nonidet P-40; $0.12 \%$ cholesteryl hemisuccinate ester; $1 \mathrm{mM}$ phenylmethylsulfonyl fluoride; $10 \mu \mathrm{g} / \mathrm{ml}$ aprotinin; $10 \mu \mathrm{g} / \mathrm{ml}$ leupeptin; $5 \mu \mathrm{g} / \mathrm{ml}$ pepstatin A; and $1 \mathrm{mM} \mathrm{DTT}$ ). For fresh human hepatocytes (Cellzdirect Inc.) and Huh7 cells, the cell pellet was collected in $400 \mu$ l of lysis buffer. Both tissue homogenates and cell lysates were then passed through a $22^{1 / 2}$-gauge needle 15 times. After centrifuging at $1,000 \mathrm{~g}$ for 10 minutes at $4^{\circ} \mathrm{C}$, the supernatant was collected and solubilized by rotating overnight at $4^{\circ} \mathrm{C}$. Lysed proteins were immunoblotted as described previously $(10,46)$ with polyclonal rabbit anti-human NPC1L1 antibody 69B (kindly provided by J.C. Cohen and H.H. Hobbs, University of Texas Southwestern Medical Center, Dallas, Texas, USA) and rabbit anti-rat RAP serum (21).

Immunohistochemistry. Acetone-fixed frozen sections of liver samples were processed for fluorescence immunohistochemistry with Bsn 4052 antibody to human NPC1L1 (2) and mouse monoclonal C219 antibody to the canalicular P-glycoprotein ABCB1 as described previously (10). Sections were examined using either a model 510 laser scanning confocal microscope or an Axioplan 2 fluorescence microscope (Zeiss).

Measurement of hepatic, plasma, and biliary lipid concentrations. Male mice $8-10$ weeks of age were fed ad libitum $(10 \mathrm{~g} / \mathrm{d})$ a synthetic low-fat, low-cholesterol diet ( $10 \%$ of energy as palm-enriched fat; $0.015 \%$ cholesterol, $w / w)$ or a low-fat, high-cholesterol diet ( $10 \%$ of energy as palm-enriched fat; $0.2 \%$ cholesterol, w/w). Gallbladder bile and blood were collected from approximately 4-hour-fasted mice that had been fed diet for 21 days. The liver was removed, weighed, and snap-frozen in liquid nitrogen. Hepatic and plasma lipid concentrations were measured as described previously $(47,48)$. To determine plasma lipoprotein cholesterol distribution, equal volumes of plasma from 6-7 mice was pooled, and lipoprotein classes were separated by gel filtration chromatography as described previously (30).

For analysis of biliary lipid concentrations, a measured volume (5-10 $\mu \mathrm{l})$ of bile was extracted with $2: 1 \mathrm{CHCl}_{3} / \mathrm{MeOH}$ in the presence of $10 \mu \mathrm{g} 5 \alpha$-cholestane. The organic phase was analyzed for cholesterol content by gas-liquid chromatography (30) and for PL content using Phospholipids B (Wako) enzymatic assay kit $(30,47)$. The aqueous phase was analyzed for BA content using an enzymatic assay employing hydroxysteroid dehydrogenase (30).

Determination of apolipoprotein compositions of plasma lipoproteins. Plasma was pooled from 6 WT and $4 \mathrm{~L} 1-\mathrm{Tg} 112$ male mice fed the $0.2 \%$ cholesterol diet for 21 days. Total plasma lipoproteins were isolated by adjusting the density of the plasma to $1.225 \mathrm{~g} / \mathrm{ml}$ with $\mathrm{KBr}$ and then centrifuging the plasma in an Optima MAX-E Ultracentrifuge (Beckman) at 356,000 $\mathrm{g}$ for 3 hours at $15^{\circ} \mathrm{C}$ using a TLA 120.2 rotor. The total lipoprotein fraction 
was separated by gel filtration chromatography using a Superose 6 column (Amersham Pharmacia Biotech) run at a flow rate of $0.5 \mathrm{ml} / \mathrm{min}$. Fractions were collected at 1-minute intervals during the chromatography step. Equal volumes $(20 \mu \mathrm{l})$ of fractions $19-32$ were mixed with $6 \mu \mathrm{l} 20 \%$ SDS $/ 100 \mathrm{mM}$ DTT, after which $6 \mu \mathrm{l} 5 \times$ sample buffer $(250 \mathrm{mM}$ Tris, $\mathrm{pH}$ 6.8; $25 \%$ glycerol; and $0.25 \%$ bromphenol blue) was added. An equal volume of each fraction was separated on $4 \%-12 \%$ polyacrylamide gradient gels (Cambrex). One set of gels was stained with $0.02 \%$ Coomassie blue in $30 \% \mathrm{MeOH} / 10 \%$ glacial acetic acid. The protein from the second set of gels was transferred to nitrocellulose membranes, which were then blocked with $5 \%$ nonfat dried milk dissolved in immunoblotting buffer $(150 \mathrm{mM} \mathrm{NaCl} ; 20 \mathrm{mM}$ Tris- $\mathrm{HCl}$, $\mathrm{pH}$ 7.4; and $0.05 \%$ Tween-20). The membranes were probed with $1 \mu \mathrm{g} / \mathrm{ml}$ goat anti-human apoB IgG (Academy Bio-Medical Co.) in incubation buffer ( $3 \%$ nonfat dried milk; $150 \mathrm{mM} \mathrm{NaCl} ; 20 \mathrm{mM}$ Tris-HCl, pH 7.4; 0.2\% Tween-20; and $0.5 \mathrm{mM} \mathrm{MgCl}_{2}$ ), washed with immunoblotting buffer, and were probed with a 1:5,000 dilution of anti-goat IgG conjugated to HRP (Sigma-Aldrich) in incubation buffer. The membranes were washed with immunoblotting buffer and treated with SuperSignal West Pico Chemiluminescent Substrate (Pierce). Visualization of apoB48 and apoB100 was achieved by exposing the membranes to X-Omat XLS-1 film (Kodak).

Quantification of plasma apoE. Plasma was immunoblotted as described above with a rabbit anti-rat apoE serum (kindly provided by J. Herz, University of Texas Southwestern Medical Center, Dallas, Texas, USA). Blots were quantified by densitometry.

Analysis of lipoprotein percent chemical composition and FA composition of the $C E$. Following fractionation by size exclusion chromatography, fractions representing LDL, ERL, and HDL were pooled. Total lipids were extracted from the samples as described above for bile, and enzymatic assays were used to determine TC, FC, TG, and PL concentrations of the samples. CE concentrations were calculated by multiplying the mass difference between TC and FC by 1.67. Protein concentrations for the samples were determined using a Lowry assay (49). The percent chemical composition was calculated by dividing the concentration of each constituent by the sum of the concentrations for all the constituents. The FA composition of the CE found on LDL, ERL, and HDL were analyzed as described previously (31).

Biliary lipid excretion following ezetimibe treatment. After being fed $0.015 \%$ cholesterol diet for 18 days, male mice were gavaged daily on days 18-21 either with $0.3 \mathrm{mg}$ ezetimibe suspended in $100 \mu \mathrm{l} 0.4 \%$ methyl cellulose or with $100 \mu \mathrm{l} 0.4 \%$ methyl cellulose alone (vehicle). On day 19, the mice were placed into cages with wire floors and individually housed in order to collect stools for 3 days, and fecal neutral sterol excretion was measured as described previously (47). Mice were maintained on the same diet during the treatment. On day 22 , mice were anesthetized with an intramuscular dose of 120 $\mathrm{mg} / \mathrm{kg}$ ketamine and $20 \mathrm{mg} / \mathrm{kg}$ xylazine, the peritoneal cavity was opened to the rib cage, and the duodenum was exposed. Through an incision in the duodenum, a cannula (polyethylene 10 tubing; ID $0.28 \mathrm{~mm}$; OD $0.61 \mathrm{~mm}$ ) containing a 0.003 -inch Teflon-coated wire was inserted through the sphincter of Oddi into the common bile duct. The cannula was tied to the bile duct, and the guide wire was removed. The mice were dosed intraperitoneally with $15 \mathrm{mg} / \mathrm{kg}$ ketamine and $1.5 \mathrm{mg} / \mathrm{kg}$ xylazine, and the viscera were wetted with saline and covered with a saline-soaked gauze pad. To maintain physiological body temperature, the mice were placed under a temperature-controlled dome. After allowing any blood and intestinal contents to be cleared from the cannula, bile was collected for 2 consecutive 15 -minute periods. Biliary lipid concentrations were measured as described above.

Statistics. Significance of differences was determined for each group of values by ANOVA (Tukey-Kramer honestly significant difference). A $P$ value less than 0.05 was considered significant.

\section{Acknowledgments}

The authors thank Tanya Paschke in our Transgenic Mouse Core Facility for pronuclear microinjection, Wei Du for immunostaining, and Ramesh Shah and Heather M. Alger for assistance with bile duct cannulation. We are indebted to Juan Francisco Miguel (Pontificia Universidad Católica de Chile, Santiago, Chile) for providing samples for our initial studies of human liver NPC1L1. We thank Paul A. Dawson for review of the manuscript. This work was supported by Intramural Funds from the Department of Pathology, Wake Forest University Health Sciences (to L. Yu), and by a Scientist Development Grant from the American Heart Association (to L. Yu). R.E. Temel was supported by a postdoctoral award from the American Heart Association. Partial support was provided by NIH grant HL-49373.

Received for publication August 15, 2006, and accepted in revised form April 10, 2007.

Address correspondence to: Liqing Yu, Department of Pathology, Section on Lipid Sciences, Wake Forest University School of Medicine, Medical Center Boulevard, Winston-Salem, North Carolina 27157-1040, USA. Phone: (336) 716-0920; Fax: (336) 716-6279; E-mail: lyu@wfubmc.edu.
1. Altmann, S.W., et al. 2004. Niemann-Pick C1 Like 1 protein is critical for intestinal cholesterol absorption. Science. 303:1201-1204.

2. Davies, J.P., Scott, C., Oishi, K., Liapis, A., and Ioannou, Y.A. 2005. Inactivation of NPC1L1 causes multiple lipid transport defects and protects against diet-induced hypercholesterolemia. J. Biol. Chem. 280:12710-12720.

3. Iyer, S.P., et al. 2005. Characterization of the putative native and recombinant rat sterol transporter Niemann-Pick C1 Like 1 (NPC1L1) protein. Biochim. Biophys. Acta. 1722:282-292.

4. Davis, H.R., Jr., et al. 2004. Niemann-Pick C1 Like 1 (NPC1L1) is the intestinal phytosterol and cholesterol transporter and a key modulator of whole-body cholesterol homeostasis. J. Biol. Chem. 279:33586-33592.

5. Davies, J.P., Levy, B., and Ioannou, Y.A. 2000. Evidence for a Niemann-pick C (NPC) gene family: identification and characterization of NPC1L1. Genomics. 65:137-145.

6. van Heek, M., et al. 2000. Comparison of the activity and disposition of the novel cholesterol absorption inhibitor, SCH58235, and its glucuronide,
SCH60663. Br. J. Pharmacol. 129:1748-1754.

7. Bays, H.E., et al. 2001. Effectiveness and tolerability of ezetimibe in patients with primary hypercholesterolemia: pooled analysis of two phase II studies. Clin. Ther. 23:1209-1230.

8. Ballantyne, C.M., Abate, N., Yuan, Z., King, T.R., and Palmisano, J. 2005. Dose-comparison study of the combination of ezetimibe and simvastatin (Vytorin) versus atorvastatin in patients with hypercholesterolemia: the Vytorin Versus Atorvastatin (VYVA) study. Am. Heart J. 149:464-473.

9. Garcia-Calvo, M., et al. 2005. The target of ezetimibe is Niemann-Pick C1-Like 1 (NPC1L1). Proc. Natl. Acad. Sci. U. S. A. 102:8132-8137.

10. Yu, L., et al. 2006. Cholesterol-regulated translocation of NPC1L1 to the cell surface facilitates free cholesterol uptake. J. Biol. Chem. 281:6616-6624.

11. Yu, L., et al. 2002. Disruption of Abcg5 and Abcg8 in mice reveals their crucial role in biliary cholesterol secretion. Proc. Natl. Acad. Sci. U. S. A. 99:16237-16242.

12. Yu, L., et al. 2002. Overexpression of ABCG5 and ABCG8 promotes biliary cholesterol secretion and reduces fractional absorption of dietary cho- lesterol. J. Clin. Invest. 110:671-680. doi:10.1172/ JCI200216001.

13. Kosters, A., et al. 2003. Relation between hepatic expression of ATP-binding cassette transporters G5 and G8 and biliary cholesterol secretion in mice. J. Hepatol. 38:710-716.

14. Plosch, T., et al. 2006. Abcg5/Abcg8-independent pathways contribute to hepatobiliary cholesterol secretion in mice. Am. J. Physiol. Gastrointest. Liver Physiol. 291:G414-G423.

15. Geuken, E., et al. 2005. Hepatic expression of ABC transporters G5 and G8 does not correlate with biliary cholesterol secretion in liver transplant patients. Hepatology. 42:1166-1174.

16. Kosoglou, T., et al. 2005. Ezetimibe: a review of its metabolism, pharmacokinetics and drug interactions. Clin. Pharmacokinet. 44:467-494.

17. Van Heek, M., et al. 1997. In vivo metabolismbased discovery of a potent cholesterol absorption inhibitor, SCH58235, in the rat and rhesus monkey through the identification of the active metabolites of SCH48461. J. Pharmacol. Exp. Ther. 283:157-163.

18. Ghosal, A., et al. 2004. Identification of human UDP-glucuronosyltransferase enzyme(s) respon- 
sible for the glucuronidation of ezetimibe (Zetia). Drug Metab. Dispos. 32:314-320.

19. Simard, C., and Turgeon, J. 2003. The pharmacokinetics of ezetimibe. Can. J. Clin. Pharmacol. 10(Suppl. A):13A-20A

20. Patrick, J.E., et al. 2002. Disposition of the selective cholesterol absorption inhibitor ezetimibe in healthy male subjects. Drug Metab. Dispos. 30:430-437.

21. Herz, J., Goldstein, J.L., Strickland, D.K., Ho, Y.K., and Brown, M.S. 1991. 39-kDa protein modulates binding of ligands to low density lipoprotein receptor-related protein/alpha 2-macroglobulin receptor. J. Biol. Chem. 266:21232-21238.

22. Botta, R., et al. 2006. TSH-Dependent expression of the LDL receptor-associated protein (RAP) in thyroid epithelial cells. Thyroid. 16:1097-1104.

23. Klett, E.L., and Patel, S.B. 2004. Biomedicine. Will the real cholesterol transporter please stand up. Science. 303:1149-1150.

24. Klett, E.L., et al. 2004. A mouse model of sitosterolemia: absence of $\mathrm{Abcg} 8$ /sterolin-2 results in failure to secrete biliary cholesterol. BMC Med. 2:5

25. Plosch, T., et al. 2004. Sitosterolemia in ABC transporter G5-deficient mice is aggravated on activation of the liver-X receptor. Gastroenterology. 126:290-300

26. Repa, J.J., et al. 2002. Regulation of ATP-binding cassette sterol transporters ABCG5 and ABCG8 by the liver X receptors alpha and beta. J. Biol. Chem. 277:18793-18800.

27. Smit, J.J., et al. 1993. Homozygous disruption of the murine mdr2 P-glycoprotein gene leads to a complete absence of phospholipid from bile and to liver disease. Cell. 75:451-462.

28. Wang, R., et al. 2001. Targeted inactivation of sister of P-glycoprotein gene (spgp) in mice results in nonprogressive but persistent intrahepatic cholestasis. Proc. Natl. Acad. Sci. U. S. A. 98:2011-2016.

29. Lee, R.G., et al. 2005. ACAT2 contributes cholesteryl esters to newly secreted VLDL, whereas LCAT adds cholesteryl ester to LDL in mice. J. Lipid Res. 46:1205-1212.

30. Lee, R.G., et al. 2004. Plasma cholesteryl esters provided by lecithin:cholesterol acyltransferase and acyl-coenzyme a:cholesterol acyltransferase 2 have opposite atherosclerotic potential. Circ. Res. 95:998-1004.
31. Furbee, J.W., Jr., Francone, O., and Parks, J.S. 2001. Alteration of plasma HDL cholesteryl ester composition with transgenic expression of a point mutation (E149A) of human LCAT. J. Lipid Res. 42:1626-1635.

32. Rigotti, A., et al. 1997. A targeted mutation in the murine gene encoding the high density lipoprotein (HDL) receptor scavenger receptor class B type I reveals its key role in HDL metabolism. Proc. Natl. Acad. Sci. U. S. A. 94:12610-12615.

33. Brundert, M., et al. 2005. Scavenger receptor class B type I mediates the selective uptake of high-density lipoprotein-associated cholesteryl ester by the liver in mice. Arterioscler. Thromb. Vasc. Biol. 25:143-148.

34. Vaisman, B.L., et al. 2001. ABCA1 overexpression leads to hyperalphalipoproteinemia and increased biliary cholesterol excretion in transgenic mice. J. Clin. Invest. 108:303-309. doi:10.1172/ JCI200112517.

35. Mahley, R.W., Weisgraber, K.H., Innerarity, T., Brewer, H.B., Jr., and Assmann, G. 1975. Swine lipoproteins and atherosclerosis. Changes in the plasma lipoproteins and apoproteins induced by cholesterol feeding. Biochemistry. 14:2817-2823.

36. Sherrill, B.C., Innerarity, T.L., and Mahley, R.W. 1980. Rapid hepatic clearance of the canine lipoproteins containing only the E apoprotein by a high affinity receptor. Identity with the chylomicron remnant transport process. J. Biol. Chem. 255:1804-1807.

37. Bersot, T.P., Mahley, R.W., Brown, M.S., and Goldstein, J.L. 1976. Interaction of swine lipoproteins with the low density lipoprotein receptor in human fibroblasts. J. Biol. Chem. 251:2395-2398.

38. Pitas, R.E., Innerarity, T.L., Arnold, K.S., and Mahley, R.W. 1979. Rate and equilibrium constants for binding of apo-E HDLc (a cholesterol-induced lipoprotein) and low density lipoproteins to human fibroblasts: evidence for multiple receptor binding of apo-E HDLc. Proc. Natl. Acad. Sci. U. S. A. 76:2311-2315.

39. Mahley, R.W., Weisgraber, K.H., Innerarity, T.L., and Windmueller, H.G. 1979. Accelerated clearance of low-density and high-density lipoproteins and retarded clearance of $\mathrm{E}$ apoprotein-containing lipoproteins from the plasma of rats after modification of lysine residues. Proc. Natl. Acad. Sci. U. S. A. 76:1746-1750.

40. Mahley, R.W., Innerarity, T.L., Weisgraber, K.B., and
Oh, S.Y. 1979. Altered metabolism (in vivo and in vitro) of plasma lipoproteins after selective chemical modification of lysine residues of the apoproteins. J. Clin. Invest. 64:743-750.

41. Zetia [package insert]. 2005. Merck/ScheringPlough Pharmaceuticals. North Wales, Pennsylvania, USA.

42. Raser, J.M., and O'Shea, E.K. 2005. Noise in gene expression: origins, consequences, and control. Science. 309:2010-2013.

43. Oude Elferink, R.P., Paulusma, C.C., and Groen, A.K. 2006. Hepatocanalicular transport defects: pathophysiologic mechanisms of rare diseases. Gastroenterology. 130:908-925.

44. Simonet, W.S., Bucay, N., Lauer, S.J., and Taylor, J.M. 1993. A far-downstream hepatocyte-specific control region directs expression of the linked human apolipoprotein E and C-I genes in transgenic mice. J. Biol. Chem. 268:8221-8229.

45. Engelking, L.J., et al. 2004. Overexpression of Insig- 1 in the livers of transgenic mice inhibits SREBP processing and reduces insulin-stimulated lipogenesis. J. Clin. Invest. 113:1168-1175. doi:10.1172/JCI200420978.

46. Yu, L., Cao, G., Repa, J., and Stangl, H. 2004. Sterol regulation of scavenger receptor class B type I in macrophages. J. Lipid Res. 45:889-899.

47. Temel, R.E., et al. 2005. Intestinal cholesterol absorption is substantially reduced in mice deficient in both ATP-binding cassette transporter A1 (ABCA1) and Acyl-CoA:Cholesterol O-acyltransferase 2 (ACAT2). J. Lipid Res. 46:2423-2431.

48. Carr, T.P., Andresen, C.J., and Rudel, L.L. 1993. Enzymatic determination of triglyceride, free cholesterol, and total cholesterol in tissue lipid extracts. Clin. Biochem. 26:39-42.

49. Lowry, O.H., Rosebrough, N.J., Farr, A.L., and Randall, R.J. 1951. Protein measurement with the Folin phenol reagent. J. Biol. Chem. 193:265-275.

50. Yu, L., et al. 2005. Expression of ABCG5 and ABCG8 Is Required for Regulation of Biliary Cholesterol Secretion. J. Biol. Chem. 280:8742-8747.

51. Timmins, J.M., et al. 2005. Targeted inactivation of hepatic Abca1 causes profound hypoalphalipoproteinemia and kidney hypercatabolism of apoA-I. J. Clin. Invest. 115:1333-1342. doi:10.1172/ JCI200523915. 